\title{
ADVANCED TECHNOLOGIES FOR DEFENSE INFORMATION SYSTEM SUPPORT
}

\author{
Juliana KARAKANEVA and Georgy PAVLOV
}

$\mathrm{P}_{\mathrm{c}}$ lanning and operational command and control (C2) of the Armed Forces is a assessing a large amount of information. It also involves situation identification and assessment, making decisions in various conditions and managing their performance. The contemporary implementation of decision making information methods and tools is considered a priority way of managing information warfare - a pursuit of effective accumulation, control and use of information and knowledge.

Some of the main aspects of C2 and its information support are ${ }^{1}$ :

- $\quad$ C2 processes and their information technology. support

- Developing and implementing management information environment support.

- Efficiency and effectiveness of the information environment.

Each stage of the management process uses various, electronically represented, information resources. The effective functioning of information environment becomes a main factor for management success.

The information quality criteria are clearly and precisely defined in the US Doctrine for Command, Control, Communications, and Computer (C4) Systems Support to Joint Operations:

* Accuracy

Information that conveys the true situation.

* Relevance

Information that applies to the mission, task, or situation at hand.

* Timeliness

Information that is available in time to make decisions. 


\section{- Usability}

Information that is in common, easily understood format and displays.

\section{* Completeness}

All necessary information required by the decision-maker.

\section{* Brevity}

Information that has only the level of detail required.

\section{* Security}

Information that has been afforded adequate protection where required.

\section{Strategy, organization, technology}

The current state of affairs poses some conceptual, organizational and technological problems for the military information systems. This paper considers the information support of management problems in two main directions: conceptual (concerning Information Strategy), and technological (concerning Information Environment).

Modern command and control information systems (CCIS) are characterized by managing and processing huge amounts of information and knowledge elements of different data types in distributed communication networks. The problems in military CCIS, however, are not caused mainly by the military structures and operations complexity, but rather by the influence of three other factors: the rapidly changing conditions regarding applied technology, the operational requirements and the various user profiles.

In most cases it is not possible to design military information systems (IS), which have an overall consistent hardware and software, for conceptual and technical implementation. Contemporary operational requirements involve continuous updates and adaptations. As a result a heterogeneous environment evolves in which $\mathrm{C} 2$ processes have to be embedded. This complicates the system's support and induces information flow interruptions.

The new requirements for multi-national command structures in actual out-of-area missions - coalition warfare, peacekeeping and peace-making missions ${ }^{2}$ - cause another problem. International missions have to be planned, prepared and executed within a short time frame and in accordance with actual needs. All this requires high flexibility and adaptability of CCIS. ${ }^{3}$

Another problem is the need for a different user-friendly profile support. An information environment has to provide direct task-oriented and problem-oriented access to the information, which is actually needed in the current operational situation. The human-machine interface is developed in modern multimedia environment. 
In order to overcome the restrictions and insufficiencies of military CCIS it is necessary to make more efficient design processes based on new information technologies.

\section{Information technology (IT)}

Information technology (IT) is applied in ways that not only contribute to more effective operations, but also reflect on the operating process so that new possibilities and perspectives arise. ${ }^{4}$

The initial application of IT was in automating the work process - substituting humans for technology that performs repetitive tasks in a more reliable way. Indeed, the various applications of the information intensive workplace extend the individual's capability to process knowledge and apply technology while gaining experience in various new ways.

The nature of work, itself, shifts from functional to cross-functional, from individual to group, from fixed procedures to alternative paths, from structural to virtual organizations, from work groups to networks of problem solvers. The transition to an information environment inevitably leads to the transformation of the activity-level work processes.

Information technology is the primary stimulant for this transformation. It is the tool of the knowledge worker. The effective integration of information technology with work processes can lead to flattened organizations, more flexible and adaptive work processes and more reliable and responsive outputs.

This is the stimulant for most reengineering projects. They are driven by a desire to capitalize on the promise of efficiency and effectiveness that are available through the better use of information technology. The information technology integration is so important because the automation of work processes provides a qualitatively better outcome. The very definition of knowledge is changed in the information intensive work environment.

\section{Scientific knowledge}

The scientific knowledge is proving to be the most valuable type nowadays. It is a structure of statistically proven and codified laws, theorems, and procedures that either have been or could be validated and verified by an independent investigator.

Through scientific methods a set of conditions and events is observed. It is represented in an analytical form, collected for consideration and processed with the use of an analytical method. The produced data is then manipulated, presented and 
interpreted so that the output can be defined as information. Information, once tested, validated, and codified becomes knowledge.

\section{Scientific methods}

Advanced Information Technologies are based on a variety of tools, methods, techniques, devices and architectures. The research-workers proposed taxonomy of scientific methods, which can be used to identify and validate requirements, build prototypes, and test and evaluate software and whole information systems. ${ }^{5}$

In particular the information science methods are presented on Figure $1 .{ }^{6}$

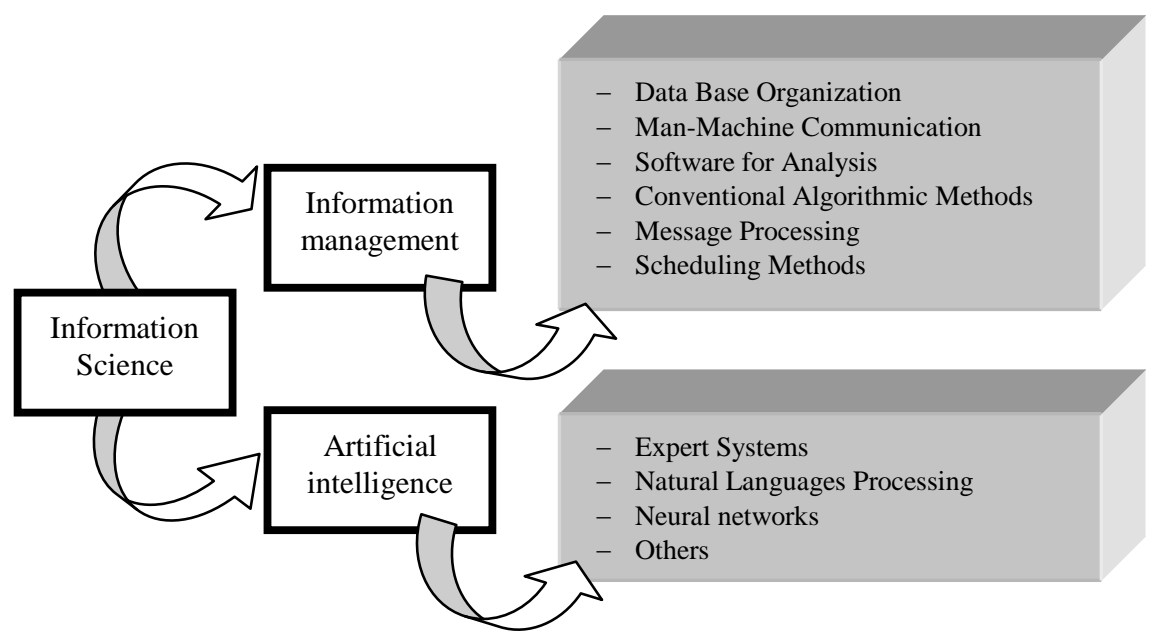

Figure 1: Relation among information science methods

For the last few years the approaches to decision support system development are knowledge based. The artificial intelligence can provide support to well-bounded problems, but it is less effective in situations with unpredictable characteristics. The latest methodology to attract attention is neural network-based models of inference making and problem solving. The neural networks are applicable to problems with characteristics that are quite different from those best suited to the artificial intelligence. They are non-deterministic, non-algorithmic, adaptive, naturally parallel, and naturally fault-tolerant powerful tool for decision making systems design. 


\section{Mission of information systems}

The classic information system aims at getting the right information to the right person at the right time. ${ }^{7}$ In today's mission environment objectives such as these are limited and short-sighted. Even the "right information" objective fails to note whether anything useful results from the delivery of the information. We suggest the following mission as appropriate for information systems: To improve the performance of people in organizations through the use of information technology.

The ultimate objective is performance improvement - an outcome or result goal instead of a go-trough-the-steps process goal. Certainly the overall organizational performance is improved, but the means of doing it are the people or groups of people that comprise the organization. The source of this improvement is the development and use of information technology, such as computers, computer software, machine readable information, and communication technologies - computer message systems, computer conferencing, or video conferencing.

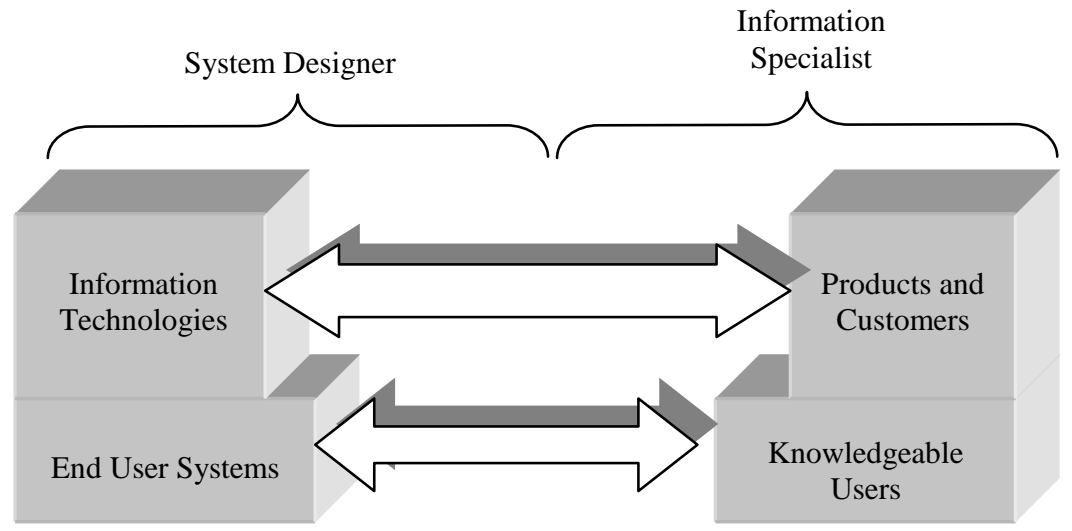

Figure 2: Relations among information experts and users

In the early days of information systems, the management support process was conducted almost entirely by a system analyst. During the past 25 years technology has become increasingly complex and powerful. At the same time the uses to which it is being applied have become increasingly sophisticated. Information systems are now viewed as system products in which users have become educated customers of the professional systems department (figure 2). The widened gap between the two boxes represents the increasingly complex process of developing and delivering the systems products. More specialization and skills are required of the system 
professionals to cover this wider gap. At the same time, many users are becoming so knowledgeable and computer-literate that they can deal with the computer directly and even develop their own systems. At the present time a certain segment of the technology is truly user-friendly, if any end-user languages to serve a wide variety of applications. Specific applications such as database inquiry report generation, and spreadsheet manipulation are regularly developed and used by managers and professionals, but it will be some time before end user will bear the majority of the system development load.

The main point of this discussion is that the technology is getting more complex, the applications are getting more sophisticated, and users are beginning to do some system development. The net result is that the management of the entire process is getting more complex and difficult while at the same time it is getting more important to do it well.

We use a model based on three principal elements:

- A set of technologies that represent products, developed by system department and other organizations.

- A set of users of the technology who can be viewed as users of these products.

- A mechanism for developing, delivering and installing these systems.

\section{Strategic Information Systems}

In this emerging age of the knowledge worker, there is a shift in the way, how managers perceive the utility of information systems. A hint of this transition can be recognized in information system labeling: some of the more systemic planning applications of management information systems are now labeled strategic information systems. These strategic information systems (SIS) refer to applications of information technology that are used to support or shape the policies and competitive strategy. Any such SIS that seeks to deliver sustained competitive advantages must be managed as a continuous innovation process - one that keeps recreating new advantages that drive the user's perception of exciting quality in new product features. There are two linkages for the innovative management of a SIS and the process of system engineering. One touch point is at the start of the technology assessment where innovation is needed to discover those unique organizational attributes that can be leveraged by information technology. This implies that the information system strategy is a product or derivative of the command and control strategy. The information system strategy focuses on those management changes that achieve a significant return through the application of information technology and that are the results of the completion of the problem-solving process where the 
technology assessment is evaluated relative to the $\mathrm{C} 2$ needs. The second touch point of SIS and systems engineering comes during the implementation process as the project team is bringing out the new work way.

A side benefit to a gradual implementation of an information system change strategy is that the change can deal more effectively with incorporation of emerging technologies. This requires the technology assessment to account for the trends in technology developments and understand how these trends may benefit the long-term change roadmap of the military organizations.

Information technology supports this with both software (GroupWare, electronic mail, and relational databases) and hardware (networks and multimedia), the most exciting innovations being GroupWare and multimedia.

\section{GroupWare and the new work way}

The way that we work has changed ever since Xerox developed a capability for drawing people together on an on-line network to share information. ${ }^{8}$ Networks first provided access for individual workers to share data and software with their local staff. Networking also increased the access of experts to information and to other problem solvers. However, there was still a boundary-individuals had to work as individuals and then merge their completed work together into a final product. GroupWare allows workgroups to cross that boundary and increase the efficiency of their shared problem-solving assets by allowing these individuals to work together in the same logical space on the computer. Because the computer has become the workplace, GroupWare allows the virtual operation of workgroup - members may be in various geographic locations and yet still share their thoughts and build their documents in a common, logical environment. Like electronic mail (Internet/ Intranet), GroupWare disrupts organizational hierarchies by permitting people to communicate with others outside their local organization. However, GroupWare differs from e-mail (Internet) in that e-mail permits one-to-one or one-to-many types of communication while GroupWare permits many-to-many communications.

The most popular type of GroupWare is represented by a single product, Lotus Notes ${ }^{\circledR}$. It combines a sophisticated messaging system with databases of work records, memoranda, and electronic documents. It changes the way that information and documents are managed in an organization. Notes ${ }^{\circledR}$ operates under a different principle for communication responsibility than does e-mail. With e-mail, the sender of the message must identify all of the individuals who need the information contained in the message. With Lotus Notes ${ }^{\circledR}$, the sender of the message forwards the message to a topical bulletin board, and anyone who needs information regarding that 
specific topic can access the bulletin board and read what associates are thinking and doing.

One report on this subject regarding the work of the staff concluded that GroupWare may be an unavoidable information processing improvement. Since GroupWare can increase the "mind share" of the corporate knowledge worker by sharing the same logical space with coworkers in a real-time operating environment, it can provide a tremendous advantage over the more linear systems used for document processing today. This equates to improvements in white-collar productivity through both cycle time reduction and increased performance through better communication.

Some of the most significant advantages listed in the report are:

- GroupWare changes the way the staff works.

- Greater access to information means that the workers must take more responsibility to access the information and to contribute to the ongoing dialogs and activities on the bulletin boards that define their work.

- GroupWare is most adaptable to a staff that has flexible cultures. Management has developed the philosophy that a decision improved and modified by group contribution is the desired outcome rather than one that may be branded as the contribution of a staff officer. There is a tendency to use GroupWare as a social bulletin board rather than as work bulletin board.

One barrier to the implementation of GroupWare is the experience that many officers have with "junk E-mail" that has to be read. For instance at one direction of MoD, a worker would get between 5 and 20 electronic messages a day. This would be in addition to the 10 to 30 telephone calls, not to mention the 5 to 20 "hard copy" mails. One thing the managers fear is that the situation only gets worse with the advent of GroupWare, which exponentially expands the available information. One way around this dilemma, however, is agent technology.

Agent technology acts like an executive secretary to help workers filter and sort through information in bulletin boards and computer files to find exactly what they need. It uses artificial intelligence and hypertext-like capabilities to identify and alert their "principal" to the presence of interesting mail.

GroupWare enables the administration to develop wholly new structures where the team is the basic building block, and the need for cross-functional knowledge and rapid response to staff demands moves organizations away from fixed hierarchies and the organization matrix. These types of teams focus on assigned objectives that require innovative solutions. Such teams operate under disciplined processes that drive for the completion of their task. Teams in this mode operate together for a period of time and then dissolve as soon as the project is completed. Examples of this 
phenomenon already exist in Research \& Development project teams, proposal generation teams, and "tiger" teams that are charted to fix a particularly perplexing problem. GroupWare helps to encourage the transition to these teams by breaking down functional barriers through an expanded access to "function-specific" information that is not allowed into the organization's common databases; creating a need for a shared language in order to communicate more effectively across functional boundaries; and providing the technical infrastructure that permits unitstaffs wide dialogue. ${ }^{9}$

\section{Conclusion}

The most important direction in enhancing the processes of command and control is the use of contemporary decision-making methodology. The environment for the information support of the management becomes crucial for success. The solution of these problems needs clearly and precisely defined management model, sound Information Strategy, and suitable organizational and technological tools. The GroupWare and the new information technologies will lead to the consolidation of all staff functions and the development of a new type of professionals, particularly in the field of strategic planning, research, and information systems.

\section{Notes:}

1 Velizar Shalamanov, "Problems of Information Security and Life Cycle Support of the Information Environment," in Proceedings of the 1996 AFCEA Sofia Seminar (Sofia: AFCEA-Sofia, 1996), pp. 22-28.

2 Yantsislav Yanakiev, Military Co-operation in South-Eastern Europe and the Future of Multinational Peace Support Operations (Rome: NATO Defence College, Spring 2000).

3 Kaster, J., A. Kaster, Componentware Approaches in Management Information Systems, HFM Workshop (The Hague: 2000).

4 Gregory H. Watson, Business Systems Engineering, Managing breakthrough changes for productivity and profit (John Wiley, 1994). 
5 Andriole, S. and Hopple, G., Inference-Making and Option Selection in Army CorpsLevel Tactical Planing: Some Hybrid Models and System Concept Storyboard (Marsdhall, VA: International Information Systems, 1987).

6 Andriole, S., Information System Design Principles for the 90s (Fairfax, Virginia: AFCEA International Press, 1990).

7 McNurlin, B., R. Sprague Jr., Information Systems Management in Practice (PrenticeHall, 1989).

8 Just as Xerox developed the WIMP or "windows-icon-mouse-pointing" computer environment, the personal computer, and object-oriented programming, the Xerox engineers also developed Ethernet as a means for connecting individual workstations together.

9 Carmel E. and J. George, "Joint Application Development (JAD) and Electronic Meeting Systems (EMS): Opportunities for the Future," Working Paper CMI WPS 91-06 (University of Arizona, Center for the Management of Information, 1991).

JULIANA KARAKANEVA is born in 1953. She hold a M.Sc. degree in Automatics and Telemechanics from the Technical University of Sofia (1976), Ph.D. degree from the Technical University of Sofia (1983) with dissertation on "Methods for Logical Design of Fail -Safe Automatic Devices for Railway Transport". Assistance-professor at the Military Research Development Institute of General Staff of Bulgarian Armed Forces, currently Defense Advanced Research Institute, "G.S.Rakovski" National Defense College. Main research interests and activities: modeling and simulations, design of command and control systems, computer assisted exercises. E - mail: ju_karakaneva@md.government.bg.

GEORGY PAVLOV is born in 1951. He hold a M.Sc. degree in Automatics and Telemechanics from the Technical University of Sofia (1974), Ph.D. degree from the Technical University of Sofia (1979) with dissertation on "Automatic Control Algorithms for Stochastic Objects". Associate-professor of Design \& Development Information systems at the Military Research Development Institute of General Staff of Bulgarian Armed Forces, currently - Defense Advanced Research Institute, "G.S.Rakovski" National Defense College. Main research interests and activities, design of command and control systems, information systems, modeling and simulations. E - mail: gpavlov@md.government.bg. 